\title{
Simulation of the control system of the electrodynamic accelerator of dust particles
}

\author{
A V Piyakov' ${ }^{1}$ D V Rodin ${ }^{1}$, M A Rodina ${ }^{1}$, A M Telegin ${ }^{1}$ and S N Kondratev ${ }^{1}$ \\ ${ }^{1}$ Samara National Research University, Moskovskoe Shosse 34A, Samara, Russia, 443086
}

\begin{abstract}
The device and control system of the accelerator of charged micron particles for simulation of micrometeorites and technogenic particles in laboratory conditions are considered. The model of the accelerator control system operation is described, the results of the accelerator control system functioning simulation for various operating modes are given. The results of comparison of mathematical simulation with experimental data are presented.
\end{abstract}

\section{Introduction}

Recently, there has been a trend of increasing concentrations of high-speed technogenic dust particles in near-Earth orbits. According to different sources, the concentration of technogenic dust particles already is three times higher than the concentration of natural origin micrometeorites. Considering the increasing demand for the reliability and durability of spacecrafts(SCs), and as well as new materials production, there is a demand for further research on the interaction of high-speed dust particles with materials of spacecrafts elements [1-3].

Currently new materials have been produced and applied in the space industry, which makes it necessary to conduct impact experiments. Conferences are regularly held on the problem of protecting spacecraft construction materials from micrometeorites. The technique of high-speed throwing is of interest not only from the point of view of modeling the factors of the space environment on the materials of spacecraft structure, but also from the point of view of feeding the thermonuclear reactors with fuel. Thus, there is an obvious need is to construct various accelerators that cover the entire range of masses of the required particles.

There are various ways to accelerate dust particles in the laboratory to simulate the interaction of micrometeorites and technogenic particles with the materials of SC construction, and to create and calibrate new micrometeorite sensors. The type and construction of the accelerator is determined by the problem posed and depends on the range of the analyzed masses and the velocities of the accelerated particles.

The main element of the dust particle accelerator is the control system, which forms accelerating voltages on the drift tubes. To solve the problem of the voltages formation accuracy, different measures can be taken: for example, in [4], the authors use the modification of a linear electrostatic accelerator, which exclude all particles with a specific charge different from 30 coulomb per kilogram from the acceleration process. Other research teams [5] use accelerators based on Van de Graaf highvoltage sources, which do not require complex control schemes, but are too complicated.

Thus, the development of high-precision control systems is essential for the construction of compact accelerators for applied research. This article concerns the operation of the electrodynamic 
accelerator of dust particles control system in nonstandard operation modes, caused by the dust contamination of the accelerator internal path.

\section{The structure of the particle accelerator}

We developed and constructed an accelerator [3, 6-8] of high speed particles, which allows to simulate impacts of micrometeorites with a size of $1 \div 10$ um and speeds of 1-15 km / s with materials of spacecraft structures under laboratory conditions.

Figure 1 shows a block diagram of a linear accelerator for simulation of micrometeorites, the path of which consists of an injector, linear electrostatic accelerator (LESA), linear electrodynamic accelerator (LEDA), three measuring lines and an experiment chamber. Vacuum in the system is provided by two pumping systems consisting of a vacuum diffusion pump AVMD -250, a forvacuum pump NVPR-16 and the necessary valves (KVE-63). The limiting residual pressure in the system is $10^{-5} \mathrm{~mm} \mathrm{Hg}$.

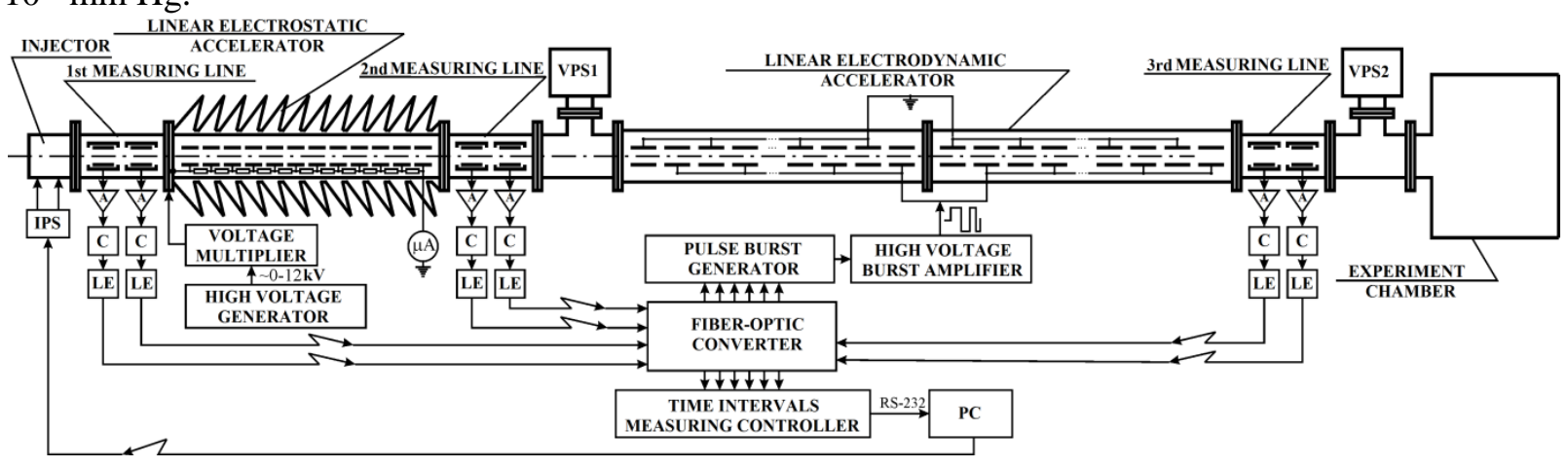

Figure 1. Structural diagram of the linear accelerator of micrometeorites. A - amplifier; C comparator; LE - laser emitter; VPS1, VPS2 - vacuum pumping system 1 and 2;

IPS- power supply of the injector.

The accelerator works as follows. The injector generates charged particles in a given mass range with the frequency of the order of $1 \mathrm{~Hz}$. The charged particle sequentially passes the first measuring line, a linear accelerator, a second measuring line, cylindrical electrodes, the third measuring line and hits the target. The first pair of measuring lines and the linear accelerator are used to determine the particle parameters (specific charge Q / $\mathrm{m}$ and initial speed V0). Flying through the measuring line, the particle induces potential to two tubes working as induction sensors. The potentioa sign is opposite to the charge of the particle. Since the tubes are made of metal, their surface is equipotential, which means it does not matter in which part to measure the voltage. According to incoming signals from the measuring line, the speed selector and the selector of specific charges form a digital code of the initial velocity of the particle at their outputs as well as the specific charge code. In the velocity selector, the time intervals of a particle path between two sensor centers for the first and second induction sensors are measured. The measured time intervals are directly proportional to the velocity of the particle. After passing through the linear accelerator, the particle receives a velocity increment. The second measuring line works similarly to the first one. According to the initial velocity and specific charge codes supplied to the variable frequency and duration pulse generator, the burst of voltage impulses is formed on its output which creates accelerating field between each pair of electrodes. This field varies in time according to the position of the particle in the accelerating path. Parameters of the burst are selected from a series of pulse-forming data loaded from a PC into the variable frequency and duration pulse generator. The variable pulse duration amplifier transmits the pulses generated in the variable frequency and duration pulse generator. The amplified pulses are applied to cylindrical electrodes. The third measuring line is connected to the interface unit and serves to obtain the output data. Then the accelerated particle hits the target and the whole process repeats. The computer produces experimental statistics and controls the accelerator dynamically. 


\section{Description of the electrodynamic accelerator control system}

To ensure the functioning of the electrodynamic part of the accelerator of micrometeorites, a control system has been developed, the functional diagram of which is shown in Figure 2.

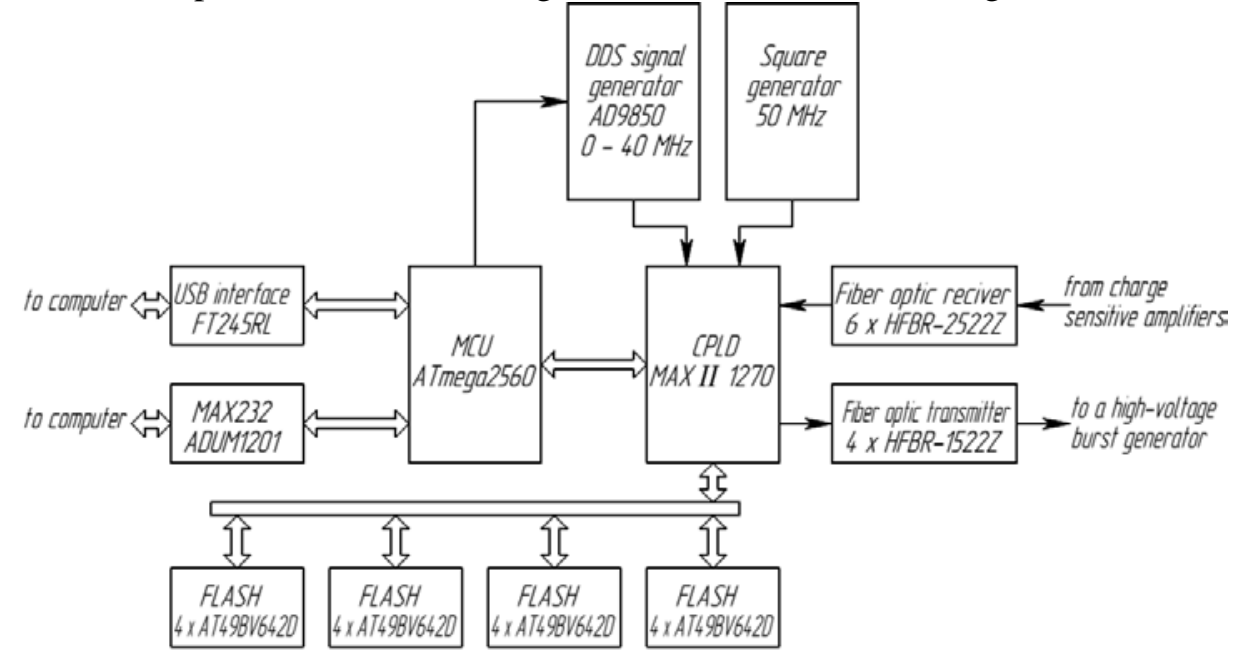

Figure 2. Functional diagram of the electrodynamic accelerator control system.

The control system works as follows. FLASH memory stores the time intervals between voltage changes on the drift tubes of a linear electrodynamic accelerator. The incoming signals from optical receivers to the CPLD MAXII 1270 contain the particle velocity before and after the linear electrostatic accelerator. The speed codes are sent to the address bus of the FLASH memory to select the desired burst of pulses corresponding to the accelerated particle. The counter implemented in CPLD starts counting clock pulses of the DDS generator. When the number of pulses on the counter coincides with the number in the FLASH memory, pulses are formed on the optical transmitters. The microcontroller serves for transferring the data about the system operation to the computer. A highspeed USB interface is used for reprogramming the memory, however, due to interference signals, this interface is not suitable for data transfer during the operation of the accelerator. Therefore, in the control system, a more noise-protected optically isolated RS-232 interface was used.

When the internal path is contaminated with metallic dust particles, the maximum accelerating voltage of the linear electrostatic accelerator is reduced. Thus, the cleaning of the accelerator path is required (Figure 3) [7].

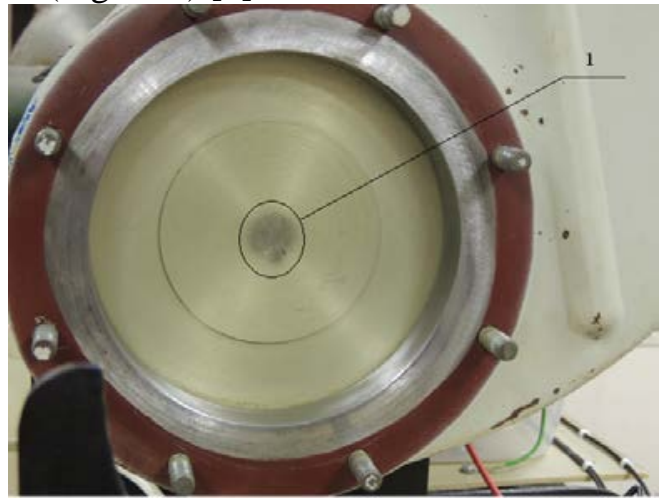

Figure 3. Disassembled accelerator path (valve in a vacuum chamber). 1 - area of microparticle dispersion in the accelerator internal path.

This time-consuming process requires disassembling the vacuum system making it necessary to provide the accelerator operation mode with a reduced accelerating voltage. This operation mode is possible when frequency of the clock generator aimed for generating pulses on the drift tubes of the electrodynamic accelerator is adjustable. For example, when operating at a voltage of $80 \mathrm{kV}$ instead of the calculated $100 \mathrm{kV}$, frequency tuning from the calculated $10 \mathrm{MHz}$ to $12.403 \mathrm{MHz}$ is required. Let us consider the results of an experiment conducted with the reduced voltage. 
Figure 4 shows the time dependence of the detection of particles on the fifth induction sensor on the specific charge of the particles. The dots represent the experimentally measured data, the solid line represents pre-calculated times embedded in the FLASH memory of the generator.

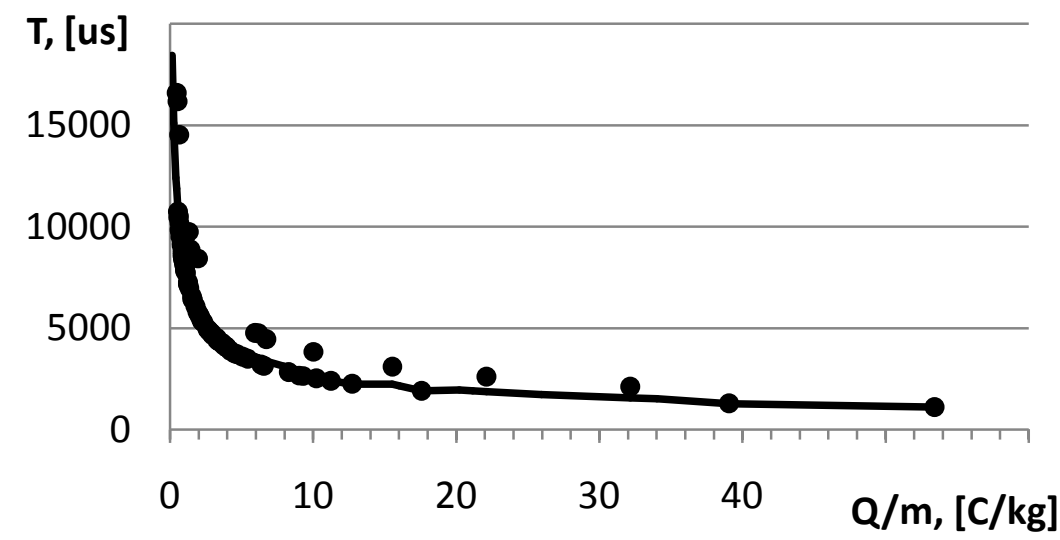

Figure 4. Dependence of the registration time of a particle on the fifth induction sensor on the specific charge.

Figure 5 shows the dependence of the particle velocity on the specific charge. Points stand for experimental data, solid line represents the calculated speed. The points that much higher or lower than the calculated curve represent the particles for which the synchronization of the accelerating pulses is lost.

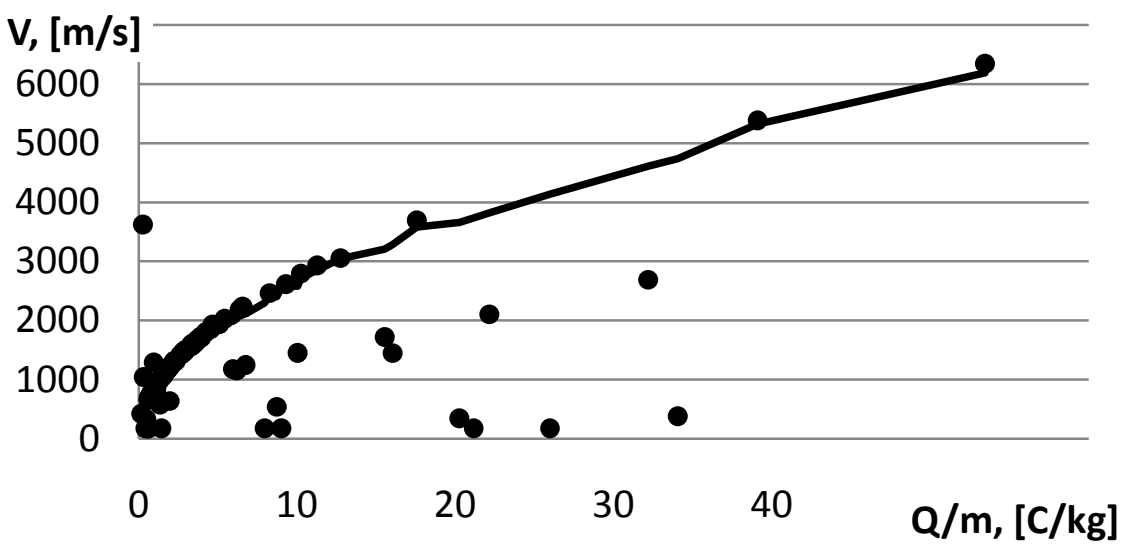

Figure 5. Dependence of the particle registration rate on the third measuring line on the specific charge.

Figures 4 and 5 show that the frequency correction with a reduced voltage of the electrostatic accelerator ensures the normal electrodynamic accelerator operation. Most of the particles pass through the internal path of the dynamic part.

\section{Results of simulation}

In order to verify the obtained experimental data, the authors modified the software described in [8], which includes:

- a class describing the state of each particle (its velocity components, coordinates, time of flight, mass-to-charge ratio values calculated for $80 \mathrm{kV}$ and $100 \mathrm{kV}$ acceleration modes);

- an array containing a two-dimensional distribution of the field in the drift tubes, calculated on the assumption that the problem is axisymmetric. The grid has a step of 9.775 $10-5 \mathrm{~m}$, which corresponds to a division of $10 \mathrm{~cm}$ of the path into 1023 intervals or 1024 nodes;

- an iterative method for calculating the trajectory of a particle, based on the Runge-Kutta algorithm. Synchronization check of the particle time of flight with the required switching times on the 
drift tubes is added, in case of inconsistency in the calculation of the current step, all field values are inverted, which results in the deceleration of the unsynchronized particles;

- the method of calculating the array with the required switching times of the voltages on the drift tubes, based on the assumption that the moment the particle is in the middle of the drift tube is the moment of commutation, taking into account the difference between the real and the measured ratio of mass to charge;

- a method for generating model packets with the Maxwellian velocity distribution corresponding to the distribution of the flux obtained in the particle injector. This method is implemented using the Box-Muller algorithm with subsequent summation of the velocity vector components and normalization for the most probable energy;

- methods for writing and reading files with particle parameters, trajectory points, and generation of header files for parallel implementation of the algorithm.

The parallel implementation of the algorithm differs singlethreaded one by storing the original matrices with particle parameters and field grid values in the form of external header files, written in plain C. The algorithm implements only the modules required directly for calculating trajectories. Multithreading was provided by connecting the MPI library. The calculation was carried out for mutually independent particles, so each involved node was used to calculate only a certain part of the trajectories.

At the initial stage, we simulated the trajectories of the real particles with the known parameters of input and output velocities and the mass to charge ratio. For each particle, the switching times were calculated, all the particles started from the center of the first drift tube with the given velocities. The particle trajectories were calculated by an iterative method, the field interpolation was carried out on the assumption that the particles have only a positive coordinate along the radial axis. For this reason, the operation of taking the module from the radial coordinate of the particle is added to the interpolator function. Interpolation was carried out for a field section of $1 \mathrm{~cm} \times 10 \mathrm{~cm}$, respectively, the $\mathrm{x}$ coordinate within the interpolator function should always lie in the range $0 \div 0.1 \mathrm{~m}$. The field interpolation operation for particles having a negative radial coordinate returned an inverted radial field component, flag of negative radial coordinate was used. The synchronous motion of the particle in the path was checked by comparing the current time of flight of the particle with the switching times from the array for the current drift tube number. The inversion of the field components in this case occurred if the time of flight was less than the smaller value or larger than the larger neighboring switching time. The intermediate points of the trajectories were stored during the transition of the centers of the drift tubes. The calculation was terminated either by a surpassing the inner radius of the tube, equal to $1 \mathrm{~cm}$, or by the transit of the center of the 40th tube. The results of the simulation are shown in Figure 6 . The experimental results are in good agreement with the calculated data, the error of calculation does not exceed 3.5\%.

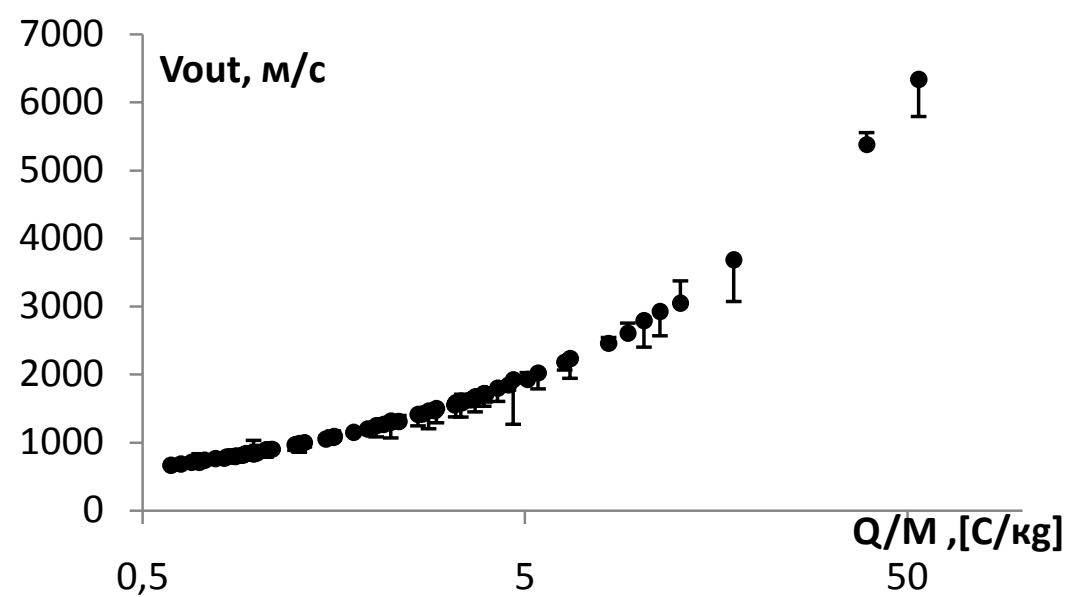

Figure 6. Comparison of experimental and model data on the velocity of the particle at the exit from the dynamic path, the absolute error bars have a scale of 10 to 1 . 
To test the performance of the acceleration system for other combinations of particle parameters, a set of 16384 particles with different initial characteristics and different specific charges was formed. Simulation of particle motion in the tract of the dynamic part of the accelerator was carried out using the implementation of the software for a personal computer and for a supercomputer Sergey Korolev. The simulation results are shown in Figures 7 and 8.
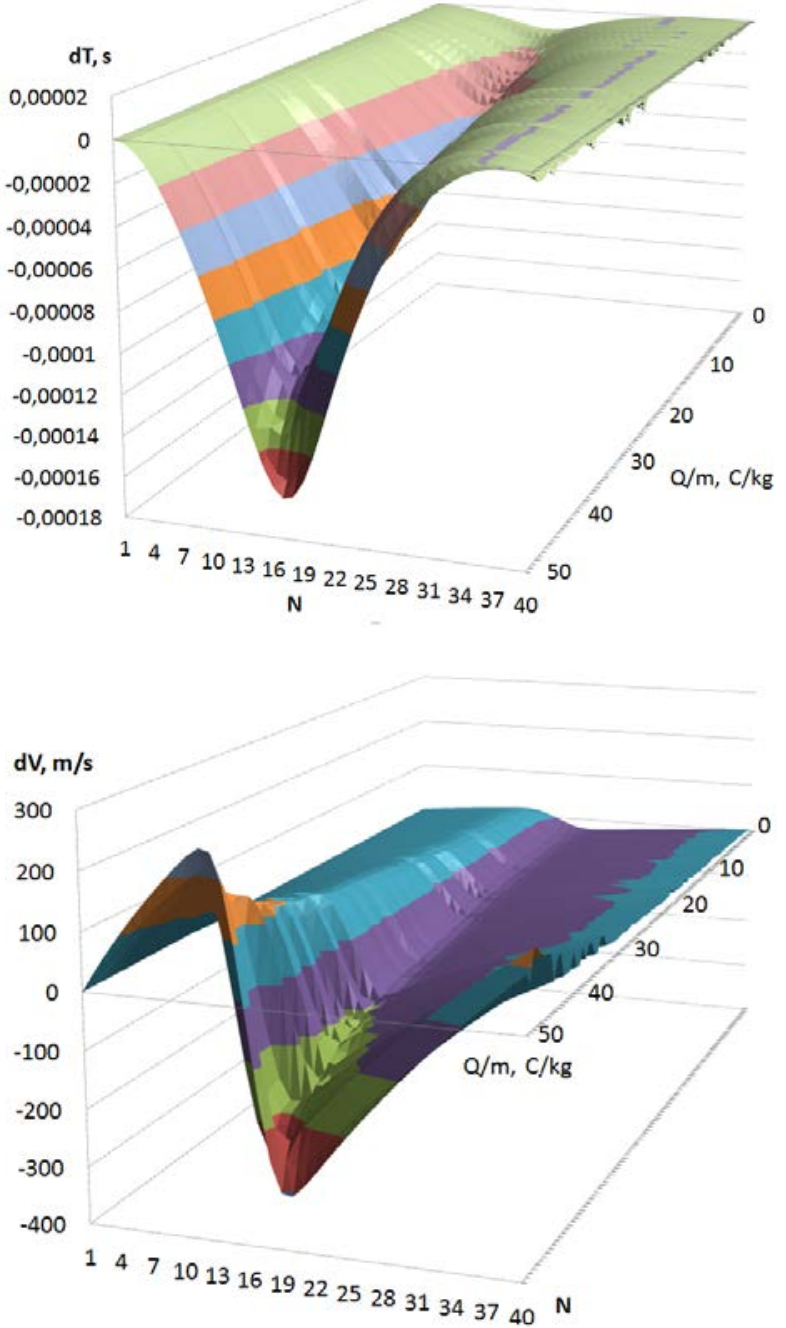

Figure 7. Difference between the calculated voltage switching times on the drift tubes of the electrodynamic accelerator, embedded in the FLASH memory of the control system and the time of particles occurrence in the centers of the drift tubes. Results are obtained by simulating the particle trajectories on the supercomputer Sergey Korolev.

Figure 8. The difference between the calculated speeds of particles during the voltage switching on the drift tubes of the electrodynamic accelerator embedded in the FLASH memory of the control system and the particle velocities during their occurrence in the centers of the drift tubes. Results are obtained by simulating particle trajectories on the supercomputer Sergey Korolev.

Figures 7 and 8 show that the first half of the path particles are slightly ahead of the calculated switching times and, as a consequence, at the initial stage they accelerated more than the calculated data suggests, but in the second half of the tract they are slowed down, and the difference between the calculated and real data is leveled.

\section{Conclusion}

Based on the results of simulation the of particles trajectories in the path of a linear electrodynamic accelerator, it can be concluded that the control system with adjustable pulse frequency of the master DDS generator from $10 \mathrm{MHz}$ to $12.403 \mathrm{MHz}$ ensures the operability of the accelerator when the real accelerating voltage of the electrostatic section deviates from the calculated one by $20 \%$. The obtained simulation results for real particles are in good agreement with experimental values, and the simulation of the system operation for a large range of input values will allow to choose the optimal switching frequency without conducting a full-scale experiment.

The implementation of software for a personal computer and supercomputer showed the same accuracy of calculations, which is explained by the choice of the same parameters of the computational grid and the time step. The version for the personal computer showed a longer 
execution time of 56 minutes 16 seconds compared to 5 minutes 46 seconds for the supercomputer, but in terms of one processor performance the first one provides a count of 1.2 million iterations per second, and the second one 740 thousand iterations per second. The loss of performance can be explained by the fact that the version for the personal computer retains all results in RAM, the same version for the supercomputer requires transferring the results of calculations between the nodes.

This problem contains a lot of mutually independent processes, so it is easy to implement the algorithm in parallel programming languages. Performance can be improved by increasing the number of computational nodes. Independence of calculating threads provides a proportional increase in performance with an increase in the number of nodes. The application of caching and preliminary processing of results on computing nodes can minimize the amount of data transferred and also increase the speed of execution.

\section{References}

[1] Semkin N D, Voronov K E and Novikov L S 2005 Registration of dust and gas particles in laboratory and space conditions (Samara: Samara State Aerospace University) p 470

[2] Raikunov G G 2014 Space debris. In 2 books. Book 1. Methods of observation and models of space debris (Moscow: FIZMATLIT) p 248

[3] Semkin N D, Kalaev M P, Telegin A M, Pijakov A V and Rodin D V 2012 Multilayer film structures under the influence of micrometeoroids and space debris Applied Physics 2 104-115

[4] Slattery J C, Becker D G, Hamermesh B and Roy N L 1973 A linear accelerator for simulated micrometeors Review of Scientific Instruments $\mathbf{4 4}$ 755-762

[5] Thomas E, Simolka J, DeLuca M, Horányi M, Janches D, Marshall R A, Munsat T, Plane J M C, and Sternovsky Z 2017 Review of Scientific Instruments 1-12

[6] Piyakov A V, Rodin D V, Rodina M A and Telegin A M 2017 Numerical simulation of motion of dust particles in an accelerator path CEUR Workshop Proceedings 1902 55-61

[7] Semkin, N D and Piyakov A V 2015 Measurements of particle distributions over the cross section of the accelerator channel for simulating micrometeorites Instruments and Experimental Techniques 58(5) 703-707

[8] Semkin, N D, Voronov K E, Piyakov A V and Piyakov I V 2009 Simulation of micrometeorites using an electrodynamical accelerator Instruments and Experimental Techniques 52(4) 595-601

[9] Semkin N D, Piyakov A V, Voronov K E, Bogoyavlenskii N L and Goryunov D V 2007 A linear accelerator for simulating micrometeorites Instruments and Experimental Techniques 50(2) $275-281$

[10] Telegin A M and Piyakov A V 2017 A study of the performance of an induction sensor for an accelerator of charged microparticles Instruments and Experimental Techniques 60(6) 875-879 